\title{
HISTORIA
}

\section{La ascendencia paterna de Daniel Carrión García}

\section{Daniel Carrión-García's paternal ancestry}

\author{
Ricardo Álvarez 1,2,3 \\ ${ }^{1}$ Instituto Nacional Materno Perinatal. Lima, Perú. \\ ${ }^{2}$ Sociedad Peruana de Patología Clínica. Lima, Perú. \\ ${ }^{3}$ Sociedad Numismática del Perú. Lima, Perú.
}

\section{Resumen}

Daniel Carrión García, mártir de la medicina peruana y héroe nacional, tenía antiguas raíces peruanas por línea materna, pero su ascendencia paterna era netamente ecuatoriana. Los Carrión fueron una de las familias más prominentes de la ciudad de Loja, a tal punto que, en 1830, al elegirse el primer Presidente de la República del Ecuador, uno de los dos candidatos fue don Manuel Carrión. La participación política de la familia continuó en los años siguientes y uno de sus miembros, el doctor José Baltazar Carrión y Torres, padre de Daniel, unió su destino al del General Juan José Flores, quien al caer en desgracia, luego de un frustrado intento revolucionario, lo arrastró consigo y debió exiliarse en el Perú. Nuestro objetivo es contribuir al conocimiento de la ascendencia paterna de Daniel Carrión García.

Palabras clave: Carrión, Daniel; Perú, historia de la medicina.

\begin{abstract}
Daniel Carrion Garcia, Peruvian medicine martyr and national hero, had ancient Peruvian roots by his Peruvian maternal line, but his father was purely Ecuadorian descent. The Carrions were one of the most prominent families in the city of Loja, to the extent that in 1830, the election of the first President of the Republic of Ecuador, one of the two candidates was Manuel Carrion. The political participation of the family continued in the following years and one of its members, Dr. Jose Baltazar Carrion y Torres, father of Daniel, joined his destiny to that of General Juan Jose Flores, who unfortunately fell in disgrace, had to go into exile and dragged the Carrions to Peru. Our goal is to contribute on the knowledge of Daniel Carrion-Garcia's paternal ancestry.
\end{abstract}

Key words: Carrion, Daniel; Peru, history of medicine.
Origen y expansión del apellido Carrión

Carrión es un apellido toponímico que surgió o resurgió en España, entre los siglos IX y $\mathrm{X}^{(1)}$. El origen del linaje corresponde al caballero Álvaro de Carreño, quien en 796 reconquistó el castillo de Monte Argel, nombre moro que luego los cristianos cambiarían por el de Carrión ${ }^{(1)}$. Allí fundó su casa solar ${ }^{(2)}$ y cambió su apellido por el de Carrión, para conmemorar este hecho de $\operatorname{armas}^{(1)}$.

Un escrito del año 912 menciona a dicha villa como Santa María de Carrión, la cual estaba surcada por el río Carrión (1). Aunque el significado de la palabra Carrión no está dilucidado, en latín medieval Carrio significaba 'dividir', 'paso' o 'vado'; por el carácter de línea divisoria que suelen tener los ríos; aquellas acepciones podrían corresponder a este vocablo ${ }^{(1)}$.

Entre 1453 y 1458, los condes de Osorno, Castañeda y Treviño juraron defender esta villa, pacto que fue ratificado en 1462 por el Consejo de los Carriones, en el que participó Alonso Martínez
Carrión, la cabeza más antigua, hasta hoy conocida, de la familia que luego se avecindaría en Ecuador y Perú ${ }^{(1)}$. Desde entonces, aquel poblado fue conocido como Carrión de los Condes ${ }^{(1)}$.

El apellido se expandió inicialmente al norte de África, luego de la caída de Granada (1492), el último bastión islámico en España. En ese período, muchas familias árabes andaluzas huyeron a Marruecos, siendo una de ellas la llamada Karrión, que siendo musulmana conservó una variación gráfica del nombre original, de raíz indudablemente española y sin ningún significado en la lengua árabe ${ }^{(3)}$.

Los Carrión llegaron a América en el siglo XVI, como consecuencia de la conquista española, y se instalaron mayoritariamente en la Audiencia de Quito. Desde antiguo existió una fluida comunicación entre el sur de Ecuador y el norte del Perú; ello permitió que el apellido se expandiera a Tumbes, Piura y Lambayeque. La separación de Guayaquil de la heredad peruana y su incorporación a la Gran Colombia no modificó estos lazos sociales, económicos y culturales.
El nacimiento de la República del Ecuador

El 9 de diciembre de 1824 se produjo la batalla de Ayacucho, en la que el ejército patriota derrotó al español, que se vio obligado a firmar la capitulación que puso fin a casi trescientos años de dominación hispana en la América meridional. Ello permitió el surgimiento de la Gran Colombia, constituida por las actuales repúblicas de Ecuador, Venezuela, Colombia y Panamá, cuya unidad era precaria y dependía en buena parte de la formidable presencia de Simón Bolívar, quien no pudo evitar que sus camaradas se enfrascaran en una lucha por el poder, que desmembró al país.

El General Juan José Flores (Figura 1), quien se distinguió por su valor durante la guerra emancipadora, había forjado una entrañable amistad con Bolívar ${ }^{(4,5)}$. Gracias a ello, en 1829, el Libertador lo nombró Prefecto General del Sur y Comandante en Jefe del Ejército ${ }^{(5)}$. Sin embargo, ocupando ese cargo, no dudó en proclamar la independencia del Distrito Sur de la Gran Colombia, el 13 de mayo 


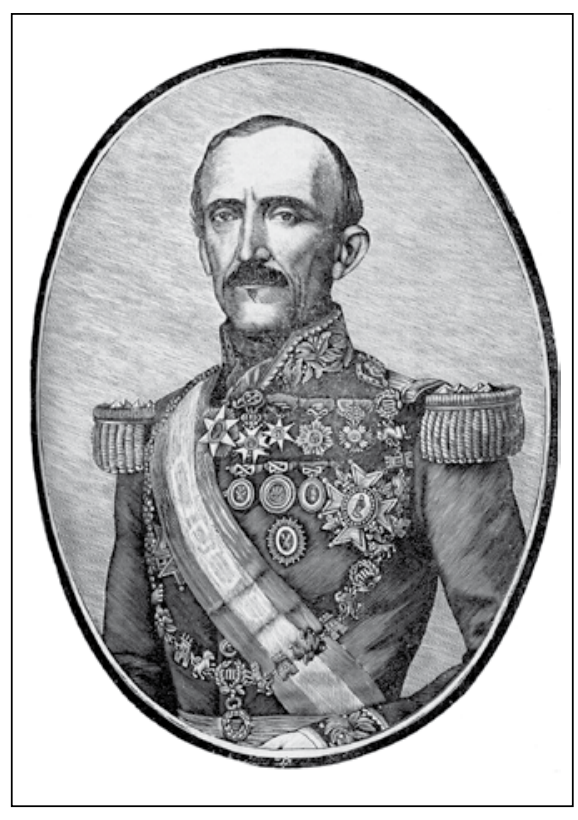

Figura 1. General Juan José Flores, Presidente de la República del Ecuador durante varios periodos de gobierno.

de 1830, y convocó a un Congreso Constituyente que se reunió en Riobamba, en agosto de ese año, dando nacimiento a la República del Ecuador.

Dicho congreso lo eligió como el primer Presidente de la República, al derrotar a su único contendor don Manuel Carrión ${ }^{(4)}$. Este último había nacido en Loja y pertenecía al mismo tronco familiar del cual sería parte años después Daniel Carrión García.

\section{Los gobiernos Flores y su incesante sed de poder}

Flores gobernó entre 1830 y 1834, en medio de dificultades políticas, sociales y económicas ${ }^{(5)}$. El 31 de enero de 1839, la Asamblea Nacional lo eligió Presidente de la República por segunda vez ${ }^{(4)}$. El 31 de marzo de 1843, dicha asamblea sancionó la nueva constitución que concedía autoridad omnímoda al poder ejecutivo; así, Flores fue elegido por tercera vez, pero ello le multiplicó sus opositores y originó el estallido de la revolución de Guayaquil, el 6 de marzo de $1845^{(4)}$.

En junio de 1845, Flores suscribió el acuerdo de 'La Virginia', por el que dejaba el poder a cambio que se respetara su rango militar y propiedades. Igualmente, aceptó exiliarse por un lapso no menor a dos años; para ese propósito, el gobierno le otorgaría 20000 Pesos (4). Sin embargo, la Asamblea Nacional revocó dicho acuerdo y Flores decidió vengarse, retomando un antiguo proyecto para instaurar una monarquía en el Ecuador, bajo la protección de España, so pretexto que el país había devenido en ingobernable ${ }^{(4)}$.

España era regentada por María Cristina, viuda de Fernando VII y madre de la futura reina Isabel II, que por entonces era menor de edad; no obstante, el poder lo tenía la cúpula militar que había derrotado a Carlos de Borbón en la guerra de sucesión española (1833-1841). Las condiciones políticas eran propicias para Flores, quien se entrevistó con María Cristina y, mientras esperaba una respuesta, adquirió armas en la Gran Bretaña y reclutó hombres en el País Vasco, Inglaterra e Irlanda ${ }^{(6)}$.

Juan Manuel Iturregui, representante peruano en Londres, intuyó que esta expedición no se limitaría al Ecuador, sino que luego avanzaría sobre el Perú; por ello protestó, junto a sus pares de Ecuador, Colombia y Chile, ante los gobiernos de España y el Reino Unido. Dichos reclamos solo merecieron evasivas, pero providencialmente nos surgirían varios aliados. Se trataba de las compañías inglesas que comerciaban en la costa del Pacífico, que temían que esta expedición alterara la tranquilidad de la región y les provocara significativas pérdidas económicas. Recién entonces, los gobiernos de España e Inglaterra intervinieron para impedirla, cuando ya se había adquirido armamento y acantonado hombres cerca del poblado español de San Sebastián ${ }^{(6)}$.

\section{Baltazar Carrión y su adhesión al general Flores}

En el Perú, durante el primer gobierno de Ramón Castilla se combatió decididamente las pretensiones de Flores, pero su sucesor, el General José Rufino Echenique (1850-1855), no dudó en apoyar al militar ecuatoriano, argumentando razones de alta geopolítica, pues Ecuador y Colombia tenían gobiernos liberales que eran un peligro para la postura con- servadora del Perú, más aún si subsistía la controversia con los colombianos sobre el pago de la deuda que contrajimos durante la guerra de emancipación ${ }^{(6)}$.

Gracias a ese apoyo, Flores arribó a Paita el 5 de julio de 1851, a bordo del bergantín chileno Almirante Bronco, que procedía de Centroamérica; cuatro días después llegó al Callao y de allí se trasladó a Lima ${ }^{(1)}$. Este proceder abiertamente sedicioso provocó que el Presidente del Ecuador, general José María Urbina, emprendiera la persecución de los partidarios del general sedicioso. Es en esas circunstancias que José Baltazar Carrión y Torres (Figura 2), quien era un fervoroso seguidor de Flores, arribó al Perú, seguramente con el propósito de reunirse con su líder ${ }^{(1)}$.

La presencia de Flores generó la protesta de los representantes de Ecuador y Colombia, pero Echenique lo acogió pretextando que se trataba de un héroe de la independencia americana. Muy pronto supo ganarse la buena voluntad de la sociedad limeña, negando reiteradamente que hubiera tenido la pretensión de establecer una monarquía en su país. Mientras tanto, continuaba con los preparativos de la expedición contra el Ecuador, que finalmente partió en abril de 1852 y luego de múltiples avatares fracasó absolutamente ${ }^{(6)}$. Este contraste obligó a Baltazar Carrión a exiliarse en el Perú.

\section{Aproximaciones a la genealogía pa- terna de Daniel Carrión García}

Es evidente que el padre de Daniel Carrión García llegó al Perú de manera fortuita, por razones eminentemente políticas. En general, los autores que biografiaron a nuestro héroe y mártir han tratado superficialmente su ascendencia paterna, debido seguramente a que la documentación y bibliografía necesaria se hallaban en el Ecuador.

En 1885, apareció la primera nota biográfica sobre Daniel Carrión, en el número 22 de la revista La Crónica Médica, fechada el 31 de octubre de aquel año ${ }^{(7)}$. Dicha nota fue muy sucinta y no refirió información alguna sobre su padre ${ }^{(7)}$. 


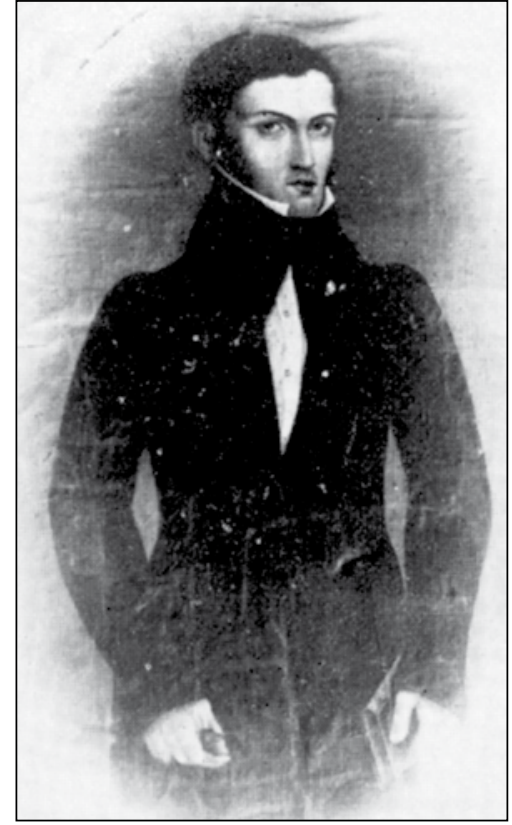

Figura 2. Doctor José Baltazar Carrión y Torres, médico y abogado ecuatoriano, padre de Daniel Carrión García.

En 1886, el gobierno del General Andrés A. Cáceres asumió los costos de la publicación de las memorias que Daniel escribió durante su enfermedad y que sus compañeros terminaron cuando ya no pudo hacerlo por sí mismo. Por primera vez, el gran público tenía acceso a este testimonio, que fue acompañado de una breve nota biográfica, que principiaba advirtiendo: "Bastante limitados, son los apuntes biográficos que podemos publicar de nuestro malogrado amigo, y no puede ser de otro modo cuando se trata de quien supo tener la abnegación suficiente para posponer su existencia en los primeros años de su vida, en bien tan solo de la humanidad y de la ciencia”. En cuanto a su ascendencia paterna, solo indicaba que "era hijo del Dr. Baltasar Carrión" (8).

En 1898, se publicó la obra La Maladie de Carrión (La Enfermedad de Carrión), del doctor Ernesto Odriozola, sin duda la investigación más completa, hasta entonces, sobre su epidemiología, cuadro clínico, diagnóstico, pronóstico y tratamiento. En ella no se incluyó una reseña biográfica de Daniel Carrión ${ }^{(9)}$.

Al asomar el siglo XX, la información sobre la ascendencia paterna de Daniel estaba circunscrita a la identidad de su padre. Desde entonces, en forma progresiva, se aportaron nuevos datos, algunos repetidos sin ninguna comprobación y, por ello, ciertos errores persistirían por muchos años.

En 1911, Hermilio Valdizán, el pionero de la historiografía médica peruana, publicó el primer tomo de su obra "La Facultad de Medicina de Lima"; allí reprodujo el brevísimo apunte biográfico sobre Daniel Carrión aparecido originalmente en La Crónica Médica ${ }^{(10)}$.

En 1921, el mencionado autor y Celso Bambarén publicaron la "Biblioteca Centenario de Medicina Peruana", en cuyo segundo tomo, dedicado a la Enfermedad de Carrión, se decía: "No intentamos la nota biográfica de Carrión; la biografía de Carrión ha sido hecha por sus amigos más queridos, por aquellos que le vieron llegar un día a la facultad de Medicina a iniciar sus estudios; por aquellos que le hallaron bueno y fuerte en la vida; por aquellos que asistieron adoloridos al cruento experimento de la inoculación y siguieron paso a paso los progresos de la enfermedad hasta la terminación fatal". Concordante con estas palabras, reprodujeron nuevamente la nota biográfica de La Crónica Médica ${ }^{(11)}$.

En 1944, Alberto Ungaro, primo materno de Daniel, señaló que don Baltazar había llegado "al Cerro de Pasco el 5 de octubre de 1853, teniendo 32 años de edad en esa fecha". En la época en que nació nuestro héroe, ya era un "hombre joven, simpático, como de unos 38 años de edad, más o menos, de mediana talla, su tipo, y fisonomía era de blanco bronceado, de pelo y barba muy negra, abundante, espesa; usaba solo bigotes que los cuidaba mucho, su voz era sonora, tenía ojos negros, expresivos y grandes; de andar ligero. El comportamiento de él y de Dolores en sociedad no era ostentoso; pero sí, tenían bastantes amistades que les guardaban afectos sinceros" (12). Ungaro dulcificó la relación entre Baltazar Carrión y Dolores García, insinuando que formaban una pareja estable y feliz, seguramente en su afán de cuidar la reputación familiar.

Agregó que Baltazar Carrión era "hijo de otro médico español llamado Francisco Carrión Riofrío", había nacido "en la Provincia de Loja, el 19 de Noviembre de 1821", y obtuvo sus títulos de médico y abogado "en las Universidades de Guayaquil - Ecuador" (12).

Añadió que don Baltazar falleció "relativamente joven, a los 44 años de edad, en la población del Distrito de Huariaca, de la Provincia de Pasco, accidentado trágicamente al escapársele una bala de su propio revolver que llevaba en el bolsillo del pantalón, en el momento en que subía sobre la montura de un caballo muy fogoso y difícil de sujetarlo. Fatalmente, cayó el arma al suelo, disparándose el plomo que le atravesó el corazón, produciéndole muerte instantánea" ${ }^{(12)}$.

"Sus restos mortales fueron sepultados el año de 1865, en el Cementerio del pueblo de Huariaca, por numerosos amigos que se aprestaron, viajando desde el Cerro de Pasco hasta dicho Distrito, con motivo de la desgracia ocurrida. El doctor Carrión vivió en la Ciudad del Cerro de Pasco, únicamente doce años, y al morir dejó a su hijo Danielito de seis años de edad" (12)

En 1949, el historiador ecuatoriano Alfredo Flores y Caamaño, nieto del General Juan José Flores ${ }^{(13)}$, publicó su investigación "El Célebre Mártir de la Ciencia Médica en el Perú", en la que afirma que "sin duda nuestro compatriota Don Baltazar, cuyos padres, podemos afirmarlo, eran también ecuatorianos de Loja, de esclarecida cepa española, arraigada allí por siglos - fue personaje de indudable valía, de temperamento enérgico, reflexivo y trabajador; inteligente y estudioso, hasta el punto de haber coronado con brillo dos carreras liberales, en especial la de Medicina; de alma noble, dispuesta a fines elevados y trascendentes; dotes singulares, resplandecientes en su unigénito, que abrazó el martirio para el bien común, y se extinguió tempranamente con la senectud del saber, la virtud de un cristiano desprendimiento y la luz acariciadora de la terrena gloria " (13).

Este es el primer autor que asevera que el abuelo paterno de Daniel Carrión era natural de la ciudad ecuatoriana de Loja, a diferencia de otros que, antes y después, afirmaron que era originario de España.

En 1957, Juan B. Lastres, uno de los principales historiadores de la medicina peruana en el siglo XX, escribió una bio- 
grafía de Daniel, con motivo del primer centenario de su nacimiento. En ella dijo textualmente: "Fue hijo del Dr. Baltazar Carrión de origen ecuatoriano". Más adelante agrega que aquel era "abogado y médico; murió trágicamente en Huariaca, en 1865, a los 44 años. Después de ejercer en el Ecuador, llegó al Cerro de Pasco donde decidió radicar en 1853" (14).

En 1971, Uriel García Cáceres, en su tesis doctoral "Historia crítica de Daniel A. Carrión y de la Medicina de su época", dice "Su padre, de quien se tiene muy pocas referencias, dícese que fue médico y abogado natural de Loja (Provincia serrana del Ecuador) y que fue exilado político en el Perú. No se conoce con exactitud si falleció cuando Daniel contaba con pocos años de edad o si retornó a su lugar de origen. Un primo de Don Baltazar Carrión, nombre del padre de Daniel, fue padrastro y tutor" ${ }^{(15)}$.

En 1973, el mismo García, refiriéndose a Baltazar Carrión, decía "no tenemos noticias concretas de la suerte que corrió después del nacimiento de su hijo Daniel. Las únicas que hemos podido encontrar están en la tesis de Peñaloza Jarrín y en un opúsculo titulado Los Carrión del autor lojano Anda. En ambos hay datos incompletos". Más adelante decía "Anda consigna que nació en 1814, se graduó en Quito de abogado en 1838, y de médico en 1840. Pidió exoneración de los derechos por su notoria pobreza y falta absoluta de recursos. El mismo autor dice que el 17 de febrero de 1844 partió hacia el Perú. Estuvo en este país por más de 10 años antes que naciera Daniel; posiblemente la mayor parte del tiempo en Cerro de Pasco" (16).

La obra del historiador y genealogista lojano don Alfonso Anda Aguirre, referida por García, lleva el título 'Los Carrión' y fue publicada por la editorial Universitaria de Loja, en 1964. En su momento fue uno de los estudios más concienzudos acerca de esta familia.

En 1985, Oscar G. Pamo reitera algunos datos que sobre el padre de Daniel proporcionaron Ungaro y Flores Caamaño, tales como su nombre, lugar y fecha de nacimiento, profesiones, y lugar, fecha y circunstancia de su muerte. En cuanto a la nacionalidad del abuelo de Daniel, coincidió con Ungaro y discordó con Flores y Caamaño ${ }^{(17)}$.

Sobre el arribo de Baltazar Carrión al Perú dice: "Corría el año de 1852, durante el gobierno del general José Rufino Echenique, y un ciudadano ecuatoriano llamado Baltazar Carrión llegó a la ciudad de Lima en condición de expatriado. El gobierno peruano le fijó como lugar de residencia la ciudad de Cerro de Pasco, ciudad minera a 4340 metros sobre el nivel del mar en nuestra sierra central, donde arribó el 5 de octubre de 1853 contando con 32 años de edad" (17).

En 2001, Gustavo Delgado publicó la obra "Daniel Alcides Carrión. Mártir de la Medicina Peruana. Héroe Nacional", en la que por primera vez un autor peruano presenta información que se remonta a varias generaciones en el árbol genealógico paterno de nuestro héroe y mártir ${ }^{(18)}$. Este autor menciona que el ancestro del padre de Daniel "se remonta a la llegada a América del primer Carrión en 1631, procedente de Sevilla (España) y fue Don Agustín Carrión Mevodis de Pacheco y Mendoza quien radicó en Cuenca (Ecuador) y contrajo matrimonio con Doña María de Alarcón” (18). Coincide con Ungaro y Flores y Caamaño en cuanto a la nacionalidad, profesiones y la condición de exiliado de Baltazar Carrión Torres ${ }^{(18)}$.

Delgado refiere que el padre de Don Baltazar era español ${ }^{(18)}$, coincidiendo con la versión de Ungaro, y discrepando con la de Flores y Caamaño.

Asimismo, Delgado proporciona interesantes datos sobre las actividades que desarrolló Baltazar Carrión durante su permanencia en Cerro de Pasco y sus alrededores. Detalla las peripecias de Alejandrina y Marco García Navarro, hermanos de la madre de Daniel, en su afán que don Baltazar cumpliera con reparar la deshonra de su pariente, y la posterior promesa de matrimonio que este formuló ante el teniente gobernador de Quiulacocha ${ }^{(18)}$. Dicha promesa nunca fue cumplida y doña Dolores quedó como madre soltera y Daniel como hijo natural.

Finalmente, tomando como referencia a Flores y Caamaño, dice "que el fallecimiento del Dr. Carrión, en 1865 , se produjo accidentalmente en Huariaca, a $35 \mathrm{~km}$ de Cerro de Pasco, en circunstancias que al cabalgar cayó su revolver y se produjo un disparo que le hirió en el pecho, de necesidad mortal. La muerte sucedió cuando él tenía 44 años de edad y su hijo Daniel contaba con ocho años" (18).

\section{Investigación de Patricio Muñoz}

La investigación del genealogista lojano Patricio Muñoz Valdivieso, en la que nos cupo colaborar, es sin duda la más documentada sobre la genealogía paterna de Daniel Carrión, develando muchas de las incógnitas subsistentes y aclarando algunos errores sostenidos por largo tiempo.

Dicho trabajo precisa el árbol genealógico desde épocas anteriores a que la familia Carrión viajara a América. En lo concerniente al presente artículo, nuestra historia empieza con don Sebastián Carrión y Merodio (1570-?), quien nació en Sevilla y casó en esa ciudad con la también sevillana doña María Pacheco de Mendoza. Se sabe que tuvieron varios hijos, entre ellos Diego y Agustín; este último es el primer ascendiente directo de Daniel Carrión en América ${ }^{(1)}$.

El Alférez don Agustín de Carrión y Merodio Pacheco (1613-1685) nació en Sevilla (España) y, animado por "una larga tradición familiar de conexiones con el nuevo mundo, emprendió la travesía del Atlántico como otros tantos parientes", siendo el primer miembro de la familia que tuvo descendencia conocida en América y tronco de los Carrión en Loja ${ }^{(1)}$. De acuerdo a los usos de aquella época, dicho personaje no utilizaba su apellido materno, por lo que en general se le conoció como Agustín de Carrión y Merodio.

Don Agustín arribó al Nuevo Mundo en 1631, como parte de la comitiva del Licenciado Juan de Valdez y Llano, sobrino del Arzobispo de Sevilla, Inquisidor General y Presidente del Consejo de Castilla, quien fue designado Oidor de la Real Audiencia de Quito. Se avecindó en Quito, por cinco años aproximadamente, y luego, en 1636, se trasladó a la villa de 
San Antonio del Cerro Rico de Zaruma, donde se dedicó a la minería. Quince años después se domicilió en la ciudad de Nuestra Señora de la Concepción de Loja, donde ocupó algunos cargos públicos ${ }^{(1)}$.

Producto de su matrimonio con María de Alarcón y Alarza, tuvo ocho hijos: Sebastián, Antonio, Mariana, Inés, Josefa, Jerónimo, Agustina y Juan. El segundo de los nombrados es el cuarto abuelo o rebisabuelo (padre del tatarabuelo) de Daniel Carrión García ${ }^{(1)}$.

El Capitán don Antonio de Carrión y Merodio Alarcón (1641-1693) nació en Loja. Contrajo matrimonio en aquella ciudad, con doña Bárbara Román y Vásquez de Vera, dama lojana que apenas frisaba los 13 años de edad. Ambos tuvieron trece hijos: José, Pedro Manuel, Diego, Agustín, Catalina, Bárbara Rosa, Antonio, Juan, Miguel, Francisco, Diego, Francisco y Gabriel. El segundo de ellos fue el tatarabuelo de Daniel Alcides Carrión ${ }^{(1)}$.

El Maestre de Campo y Capitán don Pedro Manuel de Carrión Merodio y Román (1671-1749) nació en Loja, donde ocupó varios cargos importantes, como Procurador General, Regidor y Alcalde Ordinario, este último en varias ocasiones ${ }^{(1)}$.

El domingo 15 de abril de 1703, coincidiendo con la fiesta católica de Cuasimodo, don Pedro Manuel se casó en su ciudad natal, el mismo día que sus hermanos Agustín y Antonio, con las hermanas María Rosa, Catalina y Magdalena Vaca de Vega y Arredondo, tres damas lojanas herederas del General don Jerónimo Vaca de Vega y Guevara. De este matrimonio nacieron Petronila, Josefa Francisca, Juan Francisco Agustín, Cecilia Mariana, Liberata, Melchor Domingo, Baltazara, María de la Cruz, Pastora y Pedro Crispiniano. El sexto de los mencionados fue el bisabuelo de Daniel Alcides Carrión ${ }^{(1)}$.

Don Melchor Domingo de Carrión y Vaca (1717-1771), quien ejerció el cargo de Maestre de Campo de Milicias, se casó en 1751 con su sobrina Águeda de la Piedra y Carrión, dama lojana con la que engendró diez hijos: Vicente, Eulalia Nicolasa, Pedro José Canuto,
Francisca Vicente, Agustín Victoriano, Ignacia Inés, María Josefa Liberata, María Gertrudis, Baltazara Bernardina y Juan Mariano. El tercero de ellos fue el abuelo de Daniel Alcides Carrión ${ }^{(1)}$.

Don Pedro José Canuto de Carrión y Piedra (1757-1820?) se casó con doña Victoria García Rendón y Guarnizo, el 30 de mayo de 1790, en la iglesia Sagrario de Quito. Con esta dama procreó cuatro hijos y, en 1798, luego de ocho años de matrimonio, enviudó de ella ${ }^{(1)}$.

En 1786, dicho caballero fue nombrado administrador del Real Ramo de Aguardientes en Loja, y en 1792 explotaba las vetas mineras de oro y plata situadas en Aragoto, Salacum, Guallca Rumi y Chilcapamba, en las cercanías del pueblo de Ayabaca, en Piura. En 1793, obtuvo los oficios de "Escribano de Su Majestad, Público, de Cabildo, Minas, Registros, Real Hacienda, Visitas, Residencias y Anotación de Hipotecas" ${ }^{(1)}$.

El 14 de enero de 1801 contrajo su segundo matrimonio con doña María Félix de Torres Hinojosa de la Carrera, en Cariamanga. Dicha mujer era hija de don Fernando de Torres Hinojosa y Muñoz de Urdiales y de doña Micaela de la Carrera y Carrión ${ }^{(1)}$. De este enlace nacieron trece hijos: José Manuel María, María Ignacia Jacoba, Francisca Remigia, José Francisco Melitón, José María, Pedro Andrés, María Eulalia, Tirso Antonio, María Rosa, José Baltazar, José Miguel Tomás, Pedro Manuel y María Félix Vicente. El décimo de ellos fue el padre de Daniel Alcides Carrión ${ }^{(1)}$.

Don José Baltazar de Carrión y Torres nació en Loja, el 11 de enero de 1814. Se bautizó el mismo día y fue apadrinado por Vicente de Carrión y Margarita de Carrión, quedando huérfano a los 6 años. El 13 de mayo de 1830, día en que se consagró la separación del Distrito Sur de la Gran Colombia, el joven José Baltazar, de apenas 16 años, figuró entre los firmantes del acta de emancipación. Muñoz cree que por entonces se hallaba estudiando secundaria en Quito ${ }^{(1)}$.

Cursó derecho en la Universidad Central de Quito, incorporándose como abogado el 20 de septiembre de 1838; dos años después, en 1840, se graduó de mé- dico. De regreso en Loja, logró hacerse, en corto tiempo, de una pequeña fortuna y tuvo activa participación pública en esa localidad, ocupando el rectorado del Colegio de San Bernardo (de 1841 hasta 1844 o 1845) y el cargo de Consejero $3^{3}$ ำ del municipio (1843), además del ejercicio privado de sus profesiones ${ }^{(1)}$.

En 1850, era secretario de la gobernación de Manabí; ese año se trasladó a Quito, al ser elegido diputado de dicha provincia en la Asamblea Constituyente, que se congregó hasta febrero de $1851^{(1)}$. Hacia mayo de ese año se vinculó con doña Mariana Carcelen y Barreda, viuda del Mariscal Antonio José de Sucre, y nueve años mayor que él, con la que engendró una hija llamada Mercedes Soledad ${ }^{(1)}$.

En julio de 1851, en circunstancias no precisadas, emigró al Perú por voluntad propia o desterrado; ello coincidió con la llegada del general Flores a nuestro país, por lo que es muy probable que se haya reunido con su líder ${ }^{(1)}$.

Sobre la muerte de Baltazar Carrión conjetura que "lo más probable es que en el año 1867 de su muerte, en efecto haya recibido un balazo accidental, ya sea cabalgando o al montar un caballo". Agrega que "Después de 16 años de residencia en el Perú, presintiendo que sus días se acortaban irremediablemente después de un terrible accidente que mermó notoriamente su salud, decidió retornar a su país con la intención de conocer a su primera hija; pero, apenas traspasados los límites, al estar de vuelta en su urbe natal, aunque no todavía con intención de quedarse, la muerte no le permitió continuar con su camino". Según Muñoz, Baltazar Carrión fue sepultado, el 16 de junio de 1867, en su natal Loja ${ }^{(1)}$; esta información tiene como fuente el Archivo Parroquial de El Sagrario de dicha ciudad.

\section{Epílogo}

La investigación biográfica de los grandes hombres, en muchos casos, permitirá descubrir detalles novedosos, no importa lo mucho que se les haya estudiado. Esto debe estimular a los historiadores para que perseveren en esta búsqueda. 
Gracias a ello se ha dilucidado muchas interrogantes sobre el origen ecuatoriano de la ascendencia paterna de Daniel Carrión García, héroe y mártir de la medicina peruana.

\section{AGRADECIMIENTO}

El autor desea agradecer la importante colaboración del Doctor Luís Ricardo Robles Guerrero.

\section{REFERENCIAS BIBLIOGRÁFICAS}

1. Muñoz P. El sevillano Agustín de Carrión y Merodio. Su familia en Ecuador y Perú. Quito: Sin pie de imprenta; 2007. p. 2,11,26-42,56-70,101168,169-184.

2. Schnieper J. Diccionario de Heráldica. Madrid: Editorial LIBSA; 2001. p. 116.

3. Daoud H. Las familias tetuaníes de origen andalusí [monografía en Internet]. Tarik \& Julia Banzi. 2004 [acceso 22 noviembre 2007]. Disponible en: http://www.andalus.com/tetuani_andalusian_fam. html
4. Orellana J. El Ecuador en Cien Años de Independencia 1830-1930. Tomo I. Quito: Escuela Tipográfica Salesiana; 1930. p. 23-72.

5. Andrade M. Ecuador. Próceres de la Independencia, índice alfabético de sus nombres con algunos bocetos biográficos. Quito: Tipografía y encuadernación de la Escuela de Artes y Oficios; 1909. p. 145-150.

6. Garibaldi R. La política exterior del Perú en la era de Ramón Castilla. Lima: Tarea Asociación Gráfica Educativa; 2003. p. 170-188,216-228.

7. Daniel A. Carrión. La Crónica Médica. 1885;2(22):396, 433.

8. La Verruga Peruana y Daniel A, Carrión. Lima: Imprenta del Estado; 1886. p. 9.

9. Odriozola E. La Maladie de Carrion. París: Georges Carré \& C. Naud; 1898.

10. Valdizán H. La Facultad de Medicina de Lima. 1811-1911. Lima: Sanmarti y Cía; 1911. p. 309.

11. Valdizán H, Bambarén C. Biblioteca Centenario de Medicina Peruana. Lima: Sanmarti y Cía; 1921. p. 55,65 .

12. Ungaro A. Biografía Juvenil. Daniel A. Carrión. Mártir de la Ciencia Médica. 1ra edición. Lima: La Prensa; 1944. p. 6-7,79-80

13. Flores A. El Célebre Mártir de la Ciencia Médica en el Perú. Lima: Gráfica Stylo; 1949. p. 9-10.
14. Lastres J. Daniel A. Carrión. Lima: Editorial San Marcos; 1957. p. 48.

15. García U. Historia crítica de Daniel A. Carrión y de la Medicina de su época. Tesis Doctoral. Lima, Perú. Universidad Peruana Cayetano Heredia, 1971.

16. García U. Discusión crítica sobre Daniel A. Carrión y su época. Rev Viernes Médico. 1973;24(2):178.

17. Pamo 0. Temas de la Historia Médica del Perú. Lima: Megaprint Ediciones SA; 1985. p. 17$19,54$.

18. Delgado G. Daniel Alcides Carrión. Mártir de la Medicina Peruana. Héroe Nacional. Lima: Fondo Editorial UNMSM; 2001. p. 15-21,26,27.

Manuscrito recibido el 22 de agosto de 2008 y aceptado para publicación el 25 de setiembre de 2008.

Correspondencia:

Dr. Ricardo Álvarez Carrasco

Jirón Antonio Miro Quesada 1088. Dpto. 207

Lima 1, Perú

Correo-e: ralvarezcarrasco@yahoo.com 